\title{
Coded Data Rebalancing: Fundamental Limits and Constructions
}

\author{
Prasad Krishnan, V. Lalitha, Lakshmi Natarajan
}

\begin{abstract}
Distributed databases often suffer unequal distribution of data among storage nodes, which is known as 'data skew'. Data skew arises from a number of causes such as removal of existing storage nodes and addition of new empty nodes to the database. Data skew leads to performance degradations and necessitates 'rebalancing' at regular intervals to reduce the amount of skew. We define an $r$-balanced distributed database as a distributed database in which the storage across the nodes has uniform size, and each bit of the data is replicated in $r$ distinct storage nodes. We consider the problem of designing such balanced databases along with associated rebalancing schemes which maintain the $r$-balanced property under node removal and addition operations. We present a class of $r$-balanced databases (parameterized by the number of storage nodes) which have the property of structural invariance, i.e., the databases designed for different number of storage nodes have the same structure. For this class of $r$-balanced databases, we present rebalancing schemes which use coded transmissions between storage nodes, and characterize their communication loads under node addition and removal. We show that the communication cost incurred to rebalance our distributed database for node addition and removal is optimal, i.e., it achieves the minimum possible cost among all possible balanced distributed databases and rebalancing schemes.
\end{abstract}

\section{INTRODUCTION}

Distributed data analytics engines, such as Apache Ignite [1], employ (a) a file system (such as the Hadoop File System or HDFS [2]) to distribute the data across several nodes in a cluster, and (b) a distributed computation framework (such as MapReduce) to enable parallel processing of the distributed data. Generally, in such distributed file systems, the available data is allocated to storage nodes by splitting it into a number of chunks and storing them in the nodes with some replication factor, which also functions as a protection against node failures. For instance, in HDFS, the default replication factor is 3 , which means each chunk is stored in three locations among the available nodes.

Data skew in a cluster refers to the situation in which the data stored in the nodes is not uniformly distributed. The placement of data in the storage nodes can become skewed due to various reasons [3]. New nodes may arrive whenever the client running the application requires and can afford to add them, and the newly arrived nodes clearly would start off with no data in them which results in data skew. Existing nodes may leave due to node failures, which can be common when

Dr. Krishnan and Dr. Lalitha are with the Signal Processing \& Communications Research Center, International Institute of Information Technology Hyderabad, India, email:\{prasad.krishnan, lalitha.v\}@iiit.ac.in.

Dr. Natarajan is with the Department of Electrical Engineering, Indian Institute of Technology Hyderabad, email: lakshminatarajan@iith.ac.in. nodes are run on commodity hardware. In cloud computing frameworks, a node could also be removed from a user's database if it becomes unavailable due to excessive traffic (often due to the existence of other higher priority users that it has to serve). Such node removals may result in the reduction of replication factor. Data then needs to be moved across existing nodes to reinstate the desired replication factor and this movement of data may result in data skew if not done carefully. The data-allocation protocol of the file system could also result in non-uniformity in storage across the nodes. Also, the client application may not uniformly add new data to the various nodes, preferring some nodes over others. The skew in the data placement in the storage nodes, which occurs because of such reasons, results in the imbalance of traffic handled by the various nodes. Nodes which possess a large quantity of data are forced to handle most of the traffic, and viceversa. This could further result in the creation of stragglers [4], which are nodes that act as a bottleneck to the completion of a distributed computing task.

In order to prevent data-skew, most distributed file systems employ a simple technique called data rebalancing [4]-[7]. Having detected the existence of data skew in the storage nodes based on some quantitative threshold [2], [8], a data rebalancing protocol moves the data existing in the storage nodes between them so that the data skew falls below a certain threshold. As regular rebalancing becomes a necessity whenever there is a strong data skew, the rebalancing operations can still require transfers of huge amounts of data, especially in large clusters with 100s or 1000s of nodes, which is not uncommon in the present day. Thus, the rebalancing protocol is typically implemented in such a way that the amount of communication required to balance the nodes is kept low. However, the fundamental limits of this communication problem is not yet understood, and constructions of efficient rebalancing protocols remains open.

In this work, we present a formal framework for the study of the rebalancing problem on distributed databases due to data skews arising from node removal and addition. We define the notion of an r-balanced distributed database in which each node stores an equal fraction of the data, and the data is replicated $r$ times across the nodes. Under the instance of a node removal (also, a node addition), we give a formal definition for a rebalancing scheme which maintains the $r$ replication property across balanced databases before and after the node removal (also, node addition). We define the rebalancing load as the sum of the communication load of the rebalancing schemes corresponding to both node removal 


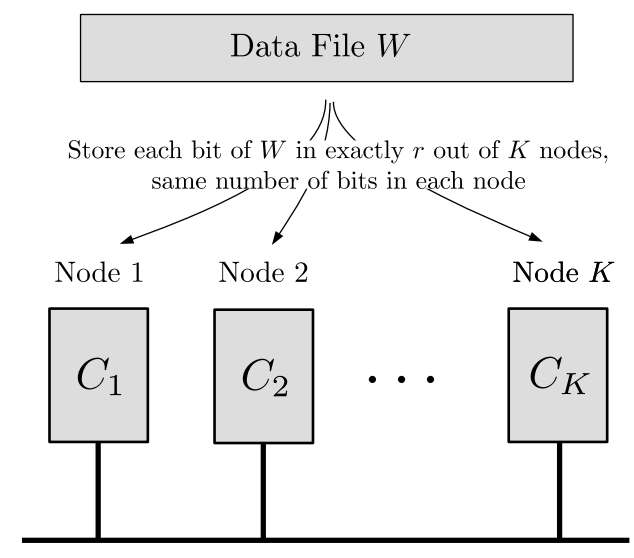

Fig. 1. An $r$-balanced distributed database across $K$ nodes. The storage nodes are connected by a shared communication link.

and addition in a distributed database, and the optimal rebalancing load $L^{*}(r)$ for a given replication factor $r$ as the minimum possible rebalancing load across all possible choices of balanced databases and rebalancing schemes. We obtain a tight characterization of $L^{*}(r)$, by deriving a lower bound on $L^{*}(r)$ and also providing an explicit construction of balanced databases and the associated rebalancing schemes with the rebalancing load equal to the presented lower bound. For the case of a node failure, the rebalancing scheme we propose uses coded transmissions, hence we call our framework Coded Data Rebalancing. The schemes we construct enable transformation of a structured database into another equivalently structured database, i.e., keeping the initial and target balanced databases within the same class. This enables the application of our distributed database and rebalancing schemes to any sequence of node removals and additions, and our scheme achieves the optimal communication load for each such sequence as long as the rebalancing operations are to be performed for each node removal or addition in the sequence.

The rest of the paper is organized as follows. In Section II. we present the system model for the rebalancing problem and the associated definitions. In Section III. we present the main result of this work (Theorem 1), which is a tight characterization of $L^{*}(r)$, and discuss its importance and implications. The next two sections, Section IV and Section $\mathrm{V}$, are devoted to the proof of Theorem 1 by showing the achievable scheme and the converse, respectively.

\section{SySTEM MODEL}

Consider a file $W$ representing the data. We assume that the file is a set of $N$ bits with the $n^{t h}$ bit denoted as $W_{n} \in\{0,1\}$, i.e., $W=\left\{W_{n}: n \in[N]\right\}$, where $[N] \triangleq\{1, \ldots, N\}$. We consider a set of $K$ nodes, indexed as $[K]=\{1, \ldots, K\}$. The nodes $[K]$ are connected to each other via a bus link. Thus there is a noiseless broadcast channel between any node $k \in[K]$ and the other nodes $[K] \backslash k$ (the set of all elements in $[K]$ except $k$ ).
A distributed database of $W$ across nodes $[K]$ (identified by labels $[K]$ ) consists of a collection $\mathcal{C}$ of subsets of $W$, $\mathcal{C}=\left\{C_{i} \subseteq W: i \in[K]\right\}$ where $C_{i}$ denotes the set of bits of $W$ stored in node $i$.

For a given distributed database $\mathcal{C}$ and a subset $B \subset[K]$, the replication factor of the $n^{\text {th }}$ bit of $W$ in $B$ is defined as $r_{n}(B)=\sum_{k \in B} \mathbb{I}\left(W_{n}\right.$ is stored in node $\left.k\right)$, where $\mathbb{I}$ denotes the indicator function.

Definition 1 ( $r$-balanced database). For an integer $r \geq 1$, an $r$-balanced distributed database on nodes $[K]$ for file $W$, denoted by $\mathcal{C}(r,[K])$, is a collection $\left\{C_{i} \subseteq W: i \in[K]\right\}$ such that (i) $r_{n}([K])=r, \forall n \in[N]$, and (ii) $\left|C_{1}\right|=\left|C_{2}\right|=$ $\ldots=\left|C_{K}\right|$. We call $r$ the replication factor of the balanced database.

For any $r$-balanced distributed database, $\sum_{k \in[K]}\left|C_{k}\right|=$ $r N$, and thus $\left|C_{k}\right|=\lambda N, \forall k \in[K]$, where $\lambda \triangleq \frac{r}{K}$. Fig. 1 illustrates the placement of the data file $W$ in an $r$-balanced distributed database.

In a given balanced distributed database on $[K]$, the addition or removal of nodes necessitates a rebalancing operation. We now formally define the rebalancing schemes associated with node removal and addition separately, along with the communication loads associated with each.

\section{A. Node Removal}

Let $k \in[K]$ be a node which is removed from the system. Let $\mathcal{C}_{k}(r,[K] \backslash k)=\left\{C_{i}(k): i \in[K] \backslash k\right\}$ be a target $r$ balanced scheme that we want to achieve in the new system consisting of nodes $[K] \backslash k$. Let $\lambda_{\text {rem }}=\frac{\lambda K}{K-1}=\frac{r}{K-1}$. In $\mathcal{C}_{k}(r,[K] \backslash k)$, for each $j$, we must have $(K-1)\left|C_{j}(k)\right|=$ $\sum_{i \in[K] \backslash k}\left|C_{i}(k)\right|=r N$. Thus, $\left|C_{j}(k)\right|=\lambda_{\text {rem }} N$.

In general, a rebalancing scheme involves each surviving storage node $i \neq k$ broadcasting a codeword $\phi_{i}\left(C_{i}\right)$ to all the other surviving nodes. At the end of these $K-1$ transmissions, each node $i \neq k$ decodes its demand using its current storage and the received codewords using a decoding function $\psi_{i}\left(C_{i},\left(\phi_{j}\left(C_{j}\right), j \in[K] \backslash\{k, i\}\right)\right)=C_{i}(k)$. Fig. 2 illustrates the data rebalancing operation when node 1 is removed from the system.

Definition 2 (Rebalancing scheme for node removal). Let $l_{i}, i \in[K] \backslash k$, be positive integers. A rebalancing scheme from $\mathcal{C}(r,[K])$ to $\mathcal{C}_{k}(r,[K] \backslash k)$ consists of a set of encoding functions

$$
\phi_{i}:\{0,1\}^{\lambda N} \rightarrow\{0,1\}^{l_{i}}, \text { for each } i \in[K] \backslash k,
$$

and a set of decoding functions

$$
\psi_{i}:\{0,1\}^{\lambda N} \times \prod_{j \in[K] \backslash\{i, k\}}\{0,1\}^{l_{j}} \rightarrow\{0,1\}^{\lambda_{\text {rem }} N},
$$

for each $i \in[K] \backslash k$, such that

$$
\psi_{i}\left(C_{i},\left(X_{j}: j \in[K] \backslash\{i, k\}\right)\right)=C_{i}(k),
$$

where $X_{j}=\phi_{j}\left(C_{j}\right)$. We denote the rebalancing scheme as $\mathcal{R}_{k, \mathcal{C}, \mathcal{C}_{k}} \triangleq\left\{\phi_{i}, \psi_{i}: i \in[K] \backslash k\right\}$. 


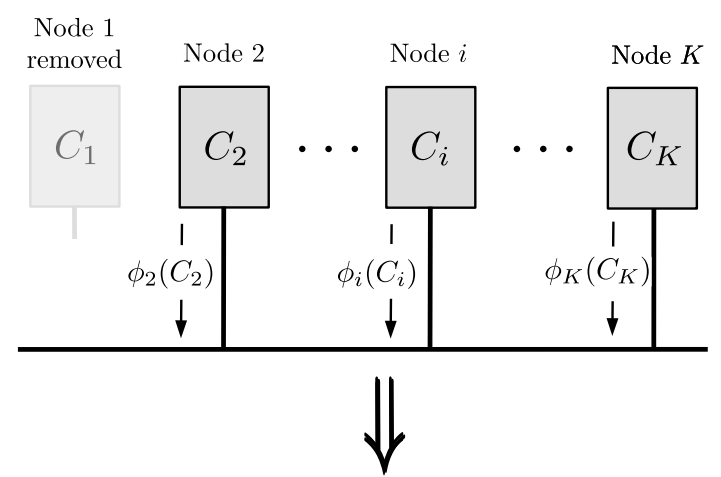

$r$-balanced database across $K-1$ nodes

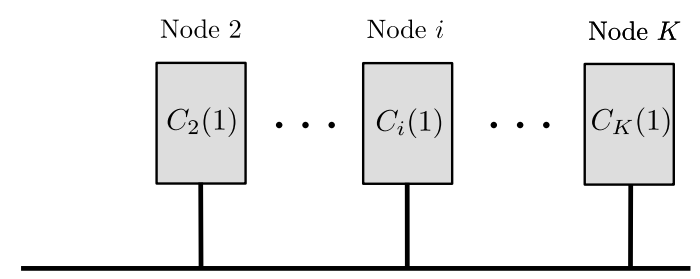

Fig. 2. Data rebalancing after node 1 is removed. Each remaining node $i$ broadcasts $\phi_{i}\left(C_{i}\right)$, and uses $C_{i}$ and the transmissions from the other $K-2$ nodes to update its contents to $C_{i}(1)$.

The communication load of such a rebalancing scheme is the total number of bits transmitted normalized by the number of bits of $C_{k}$ (the removed node's storage), given by

$$
L_{r e m}\left(\mathcal{R}_{k, \mathcal{C}, \mathcal{C}_{k}}\right) \triangleq \frac{\sum_{i \in[K] \backslash k} l_{i}}{\lambda N} .
$$

\section{B. Node Addition}

We now assume that a new node, indexed as $K+1$, is added to the system of nodes $[K]$. We assume that this node arrives without any data in its storage. Let $\mathcal{C}^{\prime}(r,[K+1])=\left\{C_{i}^{\prime}: i \in\right.$ $[K+1]\}$ be a target $r$-balanced scheme to be obtained on the set of nodes $[K+1]$. Let $\lambda_{\text {add }}=\lambda_{K+1}$. For each $j \in[K+1]$, we should have $\sum_{i \in[K+1]}\left|C_{i}^{\prime}\right|=r N=\left|C_{j}^{\prime}\right|(K+1)$, and thus $\left|C_{j}^{\prime}\right|=\lambda_{\text {add }} N=\frac{r N}{K+1}$.

We assume that each $i \in[K]$ broadcasts a codeword $\phi_{i}^{\prime}\left(C_{i}\right)$. The new node performs a decoding operation $\psi_{K+1}^{\prime}\left(\phi_{i}^{\prime}\left(C_{i}\right), i \in[K]\right)=C_{K+1}^{\prime}$ using all the transmissions. Each of the nodes $i \in[K]$ decodes its demand using $C_{i}$ and $\phi_{j}^{\prime}\left(C_{j}\right), j \in[K] \backslash\{i\}$ using its own decoding function $\psi_{i}^{\prime}\left(C_{i},\left(\phi_{j}^{\prime}\left(C_{j}\right), j \in[K] \backslash\{i\}\right)\right)=C_{i}^{\prime}$. See Fig. 3 for an illustration.

Definition 3 (Rebalancing scheme for node addition). Let $l_{i}$, $i \in[K]$, be positive integers. A rebalancing scheme from $\mathcal{C}(r,[K])$ to $\mathcal{C}^{\prime}(r,[K+1])$ consists of a set of encoding functions

$$
\phi_{i}^{\prime}:\{0,1\}^{\lambda N} \rightarrow\{0,1\}^{l_{i}}, \text { for each } i \in[K],
$$

and a set of decoding functions $\psi_{i}^{\prime}$ for each $i \in[K+1]$ defined as follows.

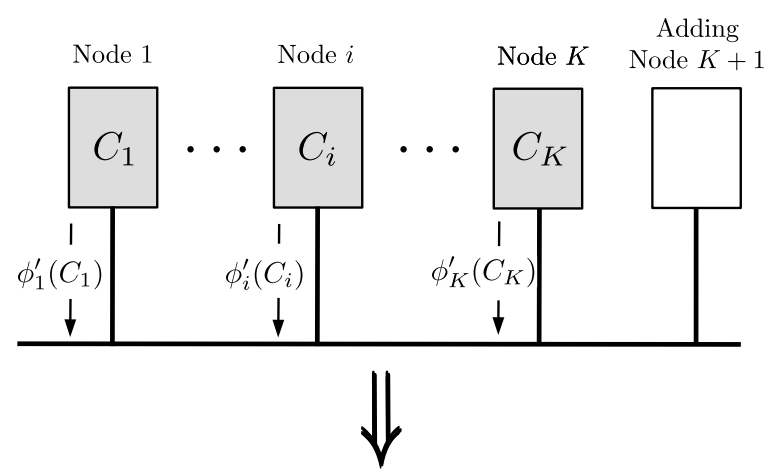

$r$-balanced database across $K+1$ nodes

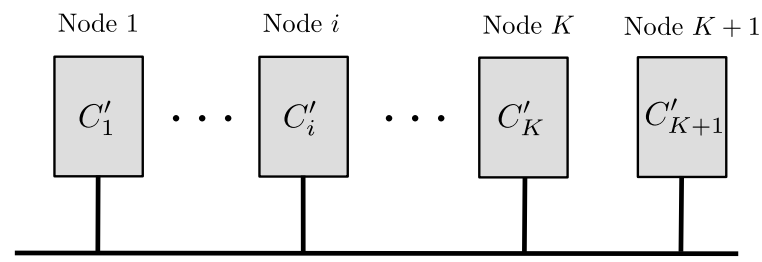

Fig. 3. Data rebalancing after an empty node $K+1$ is added. Each of the older nodes $i, 1 \leq i \leq K$, broadcasts $\phi_{i}^{\prime}\left(C_{i}\right)$ to the rest of the $K$ nodes. The new node uses these transmissions to construct its contents $C_{K+1}^{\prime}$. The older nodes modify their contents to $C_{i}^{\prime}$ using the transmissions and their current contents.

- $\psi_{i}^{\prime}:\{0,1\}^{\lambda N} \times \prod_{j \in[K] \backslash i}\{0,1\}^{l_{j}} \rightarrow\{0,1\}^{\lambda_{a d d} N}$, for each $i \in[K]$, such that

$$
\psi_{i}^{\prime}\left(C_{i},\left(X_{j}, j \in[K] \backslash\{i\}\right)\right)=C_{i}^{\prime}, \forall i \in[K]
$$

- $\psi_{K+1}^{\prime}: \prod_{j \in[K]}\{0,1\}^{l_{j}} \rightarrow\{0,1\}^{\lambda_{a d d} N}$, such that

$$
\left.\psi_{K+1}^{\prime}\left(X_{j}, j \in[K]\right)\right)=C_{K+1}^{\prime},
$$

where $X_{j}=\phi_{j}^{\prime}\left(C_{j}\right)$. We denote the rebalancing scheme as $\mathcal{R}_{\mathcal{C}, \mathcal{C}^{\prime}}^{\prime} \triangleq\left\{\phi_{i}^{\prime}, \psi_{j}^{\prime}: i \in[K], j \in[K+1]\right\}$.

The communication load of such a rebalancing scheme is the total number of bits transmitted normalized by the number of bits $\left|C_{K+1}^{\prime}\right|$ in the new node, given as

$$
L_{\text {add }}\left(\mathcal{R}_{\mathcal{C}, \mathcal{C}^{\prime}}^{\prime}\right) \triangleq \frac{\sum_{i \in[K]} l_{i}}{\lambda_{\text {add }} N} .
$$

\section{The Rebalancing Load}

Definition 4. The rebalancing load corresponding to the rebalancing schemes, $\mathcal{R}_{[K]} \triangleq\left\{\mathcal{R}_{k, \mathcal{C}, \mathcal{C}_{k}}: k \in[K]\right\}$ and $\mathcal{R}_{\mathcal{C}, \mathcal{C}^{\prime}}^{\prime}$ as given above, is defined as

$$
L\left(\mathcal{R}_{[K]}, \mathcal{R}_{\mathcal{C}, \mathcal{C}^{\prime}}^{\prime}\right) \triangleq \max _{k \in[K]} L_{r e m}\left(\mathcal{R}_{k, \mathcal{C}, \mathcal{C}_{k}}\right)+L_{a d d}\left(\mathcal{R}_{\mathcal{C}, \mathcal{C}^{\prime}}^{\prime}\right) .
$$

The optimal rebalancing load for a given replication factor $r$ is then given as the infimum of the rebalancing load (1) over all possible choices for the balanced databases and the rebalancing schemes, i.e.,

$$
L^{*}(r)=\inf _{\mathcal{C},\left\{\mathcal{C}_{k}: k \in[K]\right\}, \mathcal{C}^{\prime}} \inf _{\mathcal{R}_{[K]}, \mathcal{R}_{\mathcal{C}, \mathcal{C}^{\prime}}^{\prime}} L\left(\mathcal{R}_{[K]}, \mathcal{R}_{\mathcal{C}, \mathcal{C}^{\prime}}^{\prime}\right)
$$


Remark 1. Note that if the replication factor $r=1$, then no rebalancing scheme exists for any node removal, as the fraction of the data stored in the node being removed would be irretrievably lost. Hence we always assume that $r \geq 2$. Further, if $r=K$, then maintaining this replication factor after node removal is impossible. Hence, we assume $r \leq K-$ 1.

\section{RESULTS}

The main result of this work is a tight characterization of $L^{*}(r)$, given by the following theorem, the proof of which is given in Section $\mathrm{IV}$ and Section $\mathrm{V}$

Theorem 1. For balanced distributed databases on $K$ nodes with replication factor $r \geq 2$, the following rebalancing load $L$ is achievable

$$
L=\frac{1}{r-1}+1,
$$

if the file size $N$ is a multiple of $(r-1) P(K+1, K+1-r)$, where the symbol $P(K+1, K+1-r)$ denotes $(K+1) ! /(r !)$. Further, the above load is optimal for a given replication factor $r$, i.e., $L^{*}(r)=\frac{1}{r-1}+1$.

In order to discuss the significance of this result, we first make a few observations. Under node removal, since the initial distributed database is $r$-balanced, the number of bits whose replication factor reduces to $r-1$ after node removal is equal to $\lambda N$ (which was amount of data stored in the removed node). If naive uncoded transmissions are used to increase the replication factor of these bits to $r$, it is clear that the communication load for rebalancing under node removal will be at least 1 . Similarly we can show that the rebalancing load for node addition is also at least 1 under uncoded communications. Thus under uncoded transmissions, the rebalancing load is at least 2. However, our main result shows that the communication load for node removal can be reduced by a multiplicative factor of $r-1$ compared to uncoded schemes. Our rebalancing schemes achieve the optimal rebalancing load by careful construction of the initial and target distributed databases, which provides opportunity to perform coded transmissions for rebalancing after the node removal stage. Therefore, we refer to this paradigm of rebalancing schemes as Coded Data Rebalancing. Further, our construction also has the useful property of structural invariance, i.e., the structure of the database remains the same after node removal or addition. This facilitates the applicability of our rebalancing scheme to any sequence of node addition and node removal rebalancing operations. Since our database designs and achievability schemes for node removal and addition are optimal and depend only on the replication factor $r$, they are therefore optimal (for a suitably large file size $N$ ) for a sequence of single node removal and additions also, provided the rebalancing operation takes place after every node removal or addition.

We illustrate the coded rebalancing scheme that we propose for node removal and node addition through the following example:
Example 1. Initialization: Consider $K=5$ nodes with replication factor $r=3$. The file $W$ is partitioned into $P(K, K-r)=P(5,3)=20 \quad$ subfiles, each indexed by ordered $(K-r)=2$-sized subsets of [5]. Each storage node stores $P(K-1, K-r)=P(4,2)=12$ subfiles. Storage node $i$ stores all the subfiles not containing $i$. For instance, storage node 1

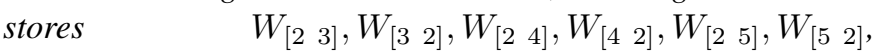
$\left.\left.\left.\left.W_{\left.\left[\begin{array}{ll}3 & 4\end{array}\right], W_{[4} 3\right]}, W_{[3} 5_{5}\right], W_{[5} 3\right], W_{[4} 5\right], W_{[5} 4\right]$.

Rebalancing and re-indexing for node removal: Consider the case when node 5 is removed. To maintain the replication factor, the contents of node 5 have to be stored among the remaining 4 nodes. The subfiles in node 5 which have their first index as $i, i=1,2,3,4$ will be stored (and hence demanded) at node $i$. In a naive uncoded scheme, the number of transmissions required $=12$ subfiles. Using coded rebalancing, we will give a scheme which uses a total of 6 subfile transmissions. In order to do this, the subfiles in node 5 are divided into 4 disjoint groups given as: $\mathcal{G}_{4}=\left\{W_{[14]}, W_{[24]}, W_{[34]}\right\}, \mathcal{G}_{3}=$

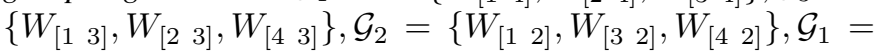
$\left.\left\{W_{\left[\begin{array}{ll}2 & 1\end{array}\right]}, W_{[3} 1\right], W_{\left[\begin{array}{ll}4 & 1\end{array}\right]}\right\}$. Each of the subfiles in a group above have the property that a subfile is present at two nodes and demanded by the remaining node. For instance,

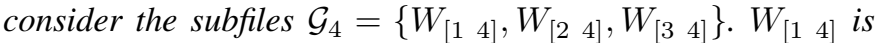
present at nodes 2,3 and demanded by node 1 . Similar is the case with other two subfiles. Now, we will show a data exchange protocol to exchange subfiles within the group. We will divide each of the three subfiles above into two parts $W_{[14]}^{(1)}, W_{[14]}^{(2)}, W_{[24]}^{(1)}, W_{[14]}^{(2)}, W_{\left[\begin{array}{ll}3 & 4\end{array}\right]}^{(1)}, W_{\left[\begin{array}{ll}3 & 4\end{array}\right]}^{(2)}$. Node $I$

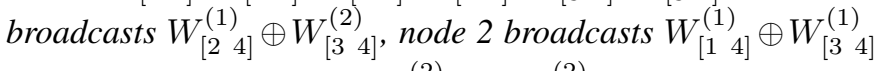

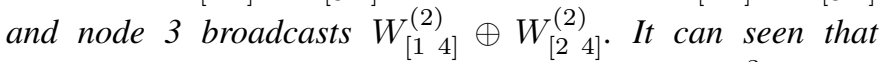
all the demands are satisfied with a total of $\frac{3}{2}$ subfile transmissions. Applying the above data exchange protocol to all the 4 groups independently, the total number of subfile transmissions required is 6 . After the above exchange, each of the 4 nodes has 15 subfiles. We will give a method to merge the subfiles and re-index them so that the re-indexing is consistent with our original initialization strategy applied to 4 nodes with 3 replicas. Storage node 1 now has the following subfiles: $\left.\left.\left.W_{[23]}, W_{[32} 2\right], W_{[24]}, W_{[42]}, \quad W_{[25]}, W_{[52]}, W_{[34} 4\right], W_{[4} 3\right]$,

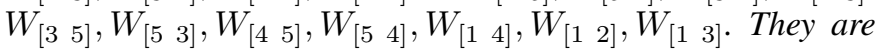
merged and re-indexed as follows:

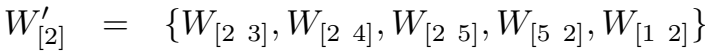

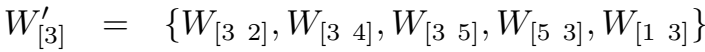

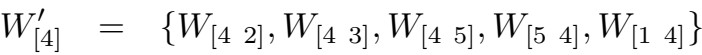

Rebalancing and re-indexing for node addition: In the case of node addition, there are three steps involved: (i) splitting the subfile (ii) transferring some splitted subfiles to the new node (iii) deleting some splitted subfiles in the original nodes. Consider the case when node 6 is added. In this case, each subfile is divided into 6 parts as follows: $\left.W_{[2} 3_{3}\right]=$

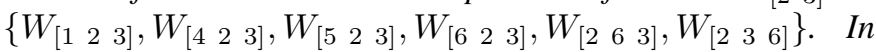




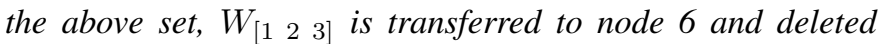
from node 1. Similar procedure is repeated for every subfile in every node. It can be seen that the re-indexing is consistent with our original initialization strategy applied to 6 nodes with 3 replicas.

\section{A. Related work}

Coded transmissions in the presence of local storage have recently been used to greatly reduce the communication load in several multi-receiver communication models, starting from [9]. This idea has since then been used in a number of similar scenarios, especially in distributed computing [10] and distributed data shuffling [11]. The framework for data rebalancing of a distributed database presented in this work enables the abstraction of a communication system with local storage and multiple receivers, and hence permits us to use coded transmissions to reduce the load of communication, similar to [9]-[11]. The results obtained in this work are therefore naturally inspired from those in these works. In particular, the achievability scheme we present is inspired from the scheme in [9] which is applicable to a cache-aided noiseless broadcast channel. Our converse proof uses arguments that are similar to those in [10].

There has been a significant amount of work related to designing erasure codes to store data in distributed file systems, which have less storage overhead and also can reconstruct data efficiently in case of node failures. In erasure coding, data is generally divided into blocks. A set of $k$ systematic blocks are used to generate $n-k$ parity blocks, where the parity blocks are linear functions of systematic blocks. The overall set of $n$ blocks comprising of $k$ systematic blocks and $n-k$ parity blocks is referred to as a stripe [12]. Upon node failure, the goal is to reconstruct the failed node using the stripes present in the existing nodes. Each stripe is processed independently and the placement of the reconstructed stripes themselves is not considered specifically, as we do in this work in the context of rebalancing. In this paper, we consider distributed file systems where data is replicated, and we consider data rebalancing operations to correct the data skew caused by both node removal and node additions.

\section{Achievability of Theorem 1}

In this section we provide construction of $r$-balanced distributed database $\mathcal{C}(r,[K])$ for storing a file across $K$ nodes for any choice of $K$ and $r$ with $2 \leq r \leq K-1$. For a given value of $r$, this family of databases is parameterized by the number of nodes $K$. We also provide accompanying data rebalancing schemes with rebalancing load $\frac{1}{r-1}+1$, such that this family of distributed databases is closed under node removal and node addition operations, see Fig 4 . That is, the node addition operation performed on the database $\mathcal{C}(r,[K])$ yields a target database for $K+1$ nodes that is equivalent to $\mathcal{C}(r,[K+1])$. Similarly any node removal operation on $\mathcal{C}(r,[K])$, irrespective of which one of the $K$ nodes is removed, yields a database that is equivalent to $\mathcal{C}(r,[K-1])$.

\section{A. A family of distributed databases}

We now describe the proposed family of distributed databases. We require the following notation to describe our construction. The symbol $P(K, l)$ denotes $K ! /(K-l)$ ! = $K(K-1) \ldots(K-(l-1))$. The $m^{\text {th }}$ component of a vector $\boldsymbol{i}=\left[\begin{array}{llll}i_{1} & \cdots & i_{l}\end{array}\right]$ will be denoted as $i_{m}$. For positive integers $l \leq K, \mathcal{S}([K], l)$ denotes the set of all vectors $i$ of length $l$ such that the components of $i$ belong to the set $[K]$ and all the components of $i$ are distinct. In other words, $\boldsymbol{i}=\left[\begin{array}{llll}i_{1} & i_{2} & \cdots & i_{l}\end{array}\right] \in \mathcal{S}([K], l)$ if and only if $i_{1}, \ldots, i_{l}$ are distinct and $\left\{i_{1}, \ldots, i_{l}\right\} \subset[K]$. For instance, the set $\mathcal{S}([3], 2)$ consists of the following six vectors

$$
\text { [1 2], [1 3], [2 1], [2 3], [3 1] and [3 2]. }
$$

Elementary arithmetic shows that $|\mathcal{S}([K], l)|=P(K, l)$. We would like the reader to think of $\boldsymbol{i} \in \mathcal{S}([K], l)$ as a subset of $[K]$ where the order in which the elements of the subset are enumerated in the vector $i$ matters. For instance, the vectors $\left[\begin{array}{lll}1 & 2 & 5\end{array}\right],\left[\begin{array}{lll}5 & 2 & 1\end{array}\right] \in \mathcal{S}([5], 3)$ arise from two different orderings of the elements of the set $\{1,2,5\}$, and these two vectors must be treated as distinct. For any element $k \in[K]$ and any vector $\boldsymbol{i} \in \mathcal{S}([K], l)$, we say that $k$ belongs to $\boldsymbol{i}$, and denote as $k \in \boldsymbol{i}$, if any one of the components of $i$ is equal to $k$. In case none of the components of $i$ is equal to $k$, we say that $k$ does not belong to $\boldsymbol{i}$ and denote this as $k \notin \boldsymbol{i}$.

The proposed distributed database scheme $\mathcal{C}(r,[K])$ : Our $r$-balanced distributed database for $K$ storage nodes is constructed as follows. As in the statement of Theorem 1 we assume that the size $N$ of the file $W$ is divisible by $(r-1) P(K+1, K+1-r)$. We partition the given file $W$ into $P(K, K-r)$ subfiles and index the subfiles using the vectors in $\mathcal{S}([K], K-r)$. The subfiles of $W$ are denoted as $W_{\boldsymbol{i}}, \boldsymbol{i} \in \mathcal{S}([K], K-r)$. We assume that the length of each of the subfiles is same, i.e., each subfile is of size $N / P(K, K-r)$ bits. The contents of the storage nodes are given by the following design

- the distributed database $\mathcal{C}(r,[K])$ stores the subfile $W_{\boldsymbol{i}}$ in storage node $k$ if and only if $k \notin \boldsymbol{i}$.

Since the length of the vector $i \in \mathcal{S}([K], K-r)$ is $K-r$, there exist exactly $r$ elements in $[K]$ that do not belong to $i$. The corresponding storage nodes, i.e., those with indices $k \notin \boldsymbol{i}$, store $W_{\boldsymbol{i}}$, and the other nodes do not store this subfile. Thus the replication factor of every subfile is precisely $r$.

The number of subfiles stored in node $k$ is equal to the size of the set $\{\boldsymbol{i} \in \mathcal{S}([K], K-r) \mid k \notin \boldsymbol{i}\}$, which is equal to $(K-1)(K-2) \cdots r=P(K-1, K-r)$. Thus, the ratio of the number of bits stored in node $k$ to the size of $W$ is

$$
\lambda=\frac{P(K-1, K-r)}{P(K, K-r)}=\frac{(K-1)(K-2) \cdots r}{K(K-1) \cdots(r+1)}=\frac{r}{K} .
$$

We conclude that $\mathcal{C}(r,[K])$ is an $r$-balanced distributed database for $K$ nodes.

Example 2. Consider $K=4$ nodes with replication factor $r=2$. The file $W$ is partitioned into $\quad P(K, K-r)=P(4,2)=12 \quad$ subfiles, each 


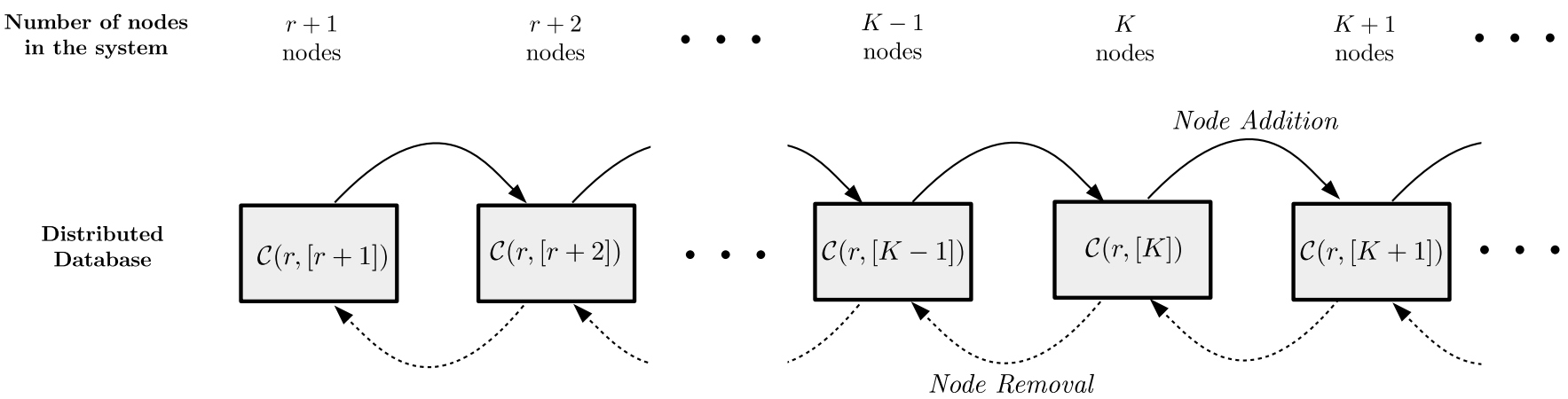

Fig. 4. The proposed family of $r$-balanced distributed databases $\mathcal{C}(r,[K]), K \geq r+1$. The databases are parameterized by the number of available storage nodes. Bold arrows and dashed arrows represent rebalancing actions applied for node addition and node removal operations, respectively. The communication loads of rebalancing for any of these node addition and node removal operations are 1 and $\frac{1}{r-1}$, respectively.

indexed by a length 2 vector in $\mathcal{S}([4], 2)$. Each storage node stores $P(K-1, K-r)=P(3,2)=6$ subfiles. For instance, storage node 1 stores

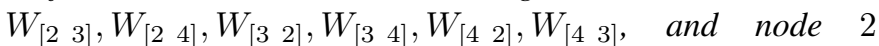
stores $W_{\left[\begin{array}{ll}1 & 3\end{array}\right]}, W_{\left[\begin{array}{ll}1 & 4\end{array}\right]}, W_{\left[\begin{array}{ll}3 & 1\end{array}\right]}, W_{\left[\begin{array}{ll}3 & 4\end{array}\right]}, W_{\left[\begin{array}{ll}4 & 1\end{array}\right]}, W_{\left[\begin{array}{ll}4 & 3\end{array}\right]}$.

\section{B. Data Rebalancing for Node Addition}

We consider the scenario where $K$ nodes are storing a file $W$ with replication factor $r$ using the distributed database scheme $\mathcal{C}(r,[K])$. A new node, denoted using the index $K+1$, is introduced into the system. This new node does not contain any information. We will now provide an algorithm to allow the $K$ pre-existing nodes to communicate with the new node in order to establish an $r$-balanced distributed database that stores the file $W$ across all $K+1$ nodes.

The proposed algorithm involves the partitioning of each subfile $W_{i}$ present in $\mathcal{C}(r,[K])$ into $K+1$ parts, and providing these parts with new indexing labels. Note that the length of $\boldsymbol{i}$ is $K-r$, and there exist $r$ elements in $[K]$, say, $j_{1}, \ldots, j_{r}$ that do not belong to $i$. Assume that $j_{1}<j_{2}<\cdots<j_{r}$. Also note that $K+1 \notin \boldsymbol{i}$, since $\boldsymbol{i} \in \mathcal{S}([K], K-r)$. Let $\boldsymbol{i}=\left[\begin{array}{llll}i_{1} & i_{2} & \cdots & i_{K-r}\end{array}\right]$. In order to partition a subfile $W_{\boldsymbol{i}}$, we split the contents of the subfile into $K+1$ equal-sized parts, and label these parts with the following length $K-r+1$ vectors (in that order)

$\left[\begin{array}{llll}j_{1} & i_{1} & \cdots & i_{K-r}\end{array}\right],\left[\begin{array}{llll}j_{2} & i_{1} & \cdots & i_{K-r}\end{array}\right], \cdots,\left[\begin{array}{llll}j_{r} & i_{1} & \cdots & i_{K-r}\end{array}\right]$, $\left[\begin{array}{llll}K+1 & i_{1} & \cdots & i_{K-r}\end{array}\right],\left[\begin{array}{lllll}i_{1} & K+1 & i_{2} & \cdots & i_{K-r}\end{array}\right], \cdots$,

$\left[\begin{array}{lllll}i_{1} & i_{2} & \cdots & i_{K-r} & K+1\end{array}\right]$.

The first $r$ of the above vectors are obtained by prefixing $i$ with $j_{1}, \ldots, j_{r}$, respectively. The remaining $K-r+1$ vectors are obtained by all possible insertions of the component $K+1$ into the vector $i$. These new parts are denoted by $W_{\left.\left[\begin{array}{llll}j_{1} & i_{1} & \cdots & i_{K-r}\end{array}\right], \ldots, W_{\left[i_{1}\right.} i_{2} \cdots i_{K-r} K+1\right]}$, respectively. Note that the new indices are vectors from the set $\mathcal{S}([K+1], K-$ $r+1)$.

The size of each of these $K+1$ new subfiles is

$$
\frac{N}{P(K, K-r)} \times \frac{1}{K+1}=\frac{N}{P(K+1, K+1-r)} \text { bits. }
$$

The data rebalancing scheme for node addition is as follows. For each $k \in[K]$, the node $k$ partitions each of the subfiles available to it into $K+1$ parts using the technique described in the previous paragraph. The subfiles available at node $k$ are $W_{\boldsymbol{i}}, \boldsymbol{i} \in \mathcal{S}([K], K-r)$ and $k \notin \boldsymbol{i}$. Note that when $W_{\boldsymbol{i}}$ is partitioned by node $k$, one of the resulting parts will have the index $\left[\begin{array}{ll}k & i\end{array}\right]$. After partitioning, node $k$ transfers the following parts to the new node

$$
W_{[k i]}, \text { where } \boldsymbol{i} \in \mathcal{S}([K], K-r) \text { and } k \notin \boldsymbol{i},
$$

while removing them from its own memory. The rest of the new parts are stored in node $k$. Node $K+1$ receives all such transmissions from each of the $K$ pre-existing nodes, and stores the received contents in its memory. It is not difficult to observe that the parts remaining in node $k$ correspond to the set of indices $\left\{\boldsymbol{i}^{\prime} \in \mathcal{S}([K+1], K-r+1) \mid k \notin \boldsymbol{i}^{\prime}\right\}$, and the parts now stored in node $K+1$ have the indices in the set $\left\{\boldsymbol{i}^{\prime} \in \mathcal{S}([K+1], K-r+1) \mid K+1 \notin \boldsymbol{i}^{\prime}\right\}$. This placement of contents of $W$ across $K+1$ nodes is identical to the distributed database scheme $\mathcal{C}(r,[K+1])$.

The number of parts communicated by node $k$ to node $K+1$ is $P(K-1, K-r)$, and the number of parts remaining with node $k$ is $K \times P(K-1, K-r)=P(K, K-r+1)$. Using (2), the fraction of the overall file stored in each of the $K+1$ nodes after data rebalancing for node addition is

$$
\lambda_{a d d}=\frac{P(K, K-r+1)}{P(K+1, K-r+1)}=\frac{r}{K+1}=\lambda \frac{K}{K+1},
$$

which is as required for an $r$-balanced scheme. The total number of bits communicated during rebalancing is

$$
K \times \frac{P(K-1, K-r) N}{P(K+1, K-r+1)}=\frac{r N}{(K+1)}=\lambda_{a d d} N .
$$

Thus, the communication load of this rebalancing scheme for node addition is $L_{a d d}=1$.

\section{Coded Data Rebalancing for Node Removal}

We now provide a rebalancing scheme for mitigating data skew when one of the nodes in the $r$-balanced distributed 
database $\mathcal{C}(r,[K])$ fails. The subfiles that were originally available in the failed node are now replicated only $(r-1)$ times in the surviving nodes. The main objective of our data balancing scheme is to place a new copy of any such subfile $W_{i}$ in the node with index $i_{1}$, where $i_{1}$ is the first component of $i$. This will ensure that all subfiles are replicated $r$ times across $(K-1)$ nodes. The subfiles are then combined in a specific way and re-indexed so that the resulting database is structurally identical to $\mathcal{C}(r,[K-1])$.

1) Review of a Data Exchange Protocol: As one of the components of our data rebalancing scheme, we make use of a communication efficient protocol for exchanging data between a group of $r$ storage nodes. This protocol is used when $r$ nodes are connected by a common broadcast link, each node stores a distinct $(r-1)$ subset of a set of $r$ files $B_{1}, \ldots, B_{r}$, and each node demands the unique file that is not available in its memory. If the size of each of the files $B_{1}, \ldots, B_{r}$ is $\ell$ bits, the overall communication cost of this protocol, i.e., the total number of bits broadcast by all the $r$ nodes, is $\ell r /(r-1)$ bits. This protocol is known in the literature; for instance, [13] uses this for coded MapReduce. For the sake of completeness, we provide a brief description of this protocol in Appendix A.

2) Data Rebalancing: Assume that an arbitrary node $k$ is removed from the distributed database $\mathcal{C}(r,[K])$. We denote the index set of the remaining nodes as $\overline{\mathcal{K}}=[K] \backslash k$. Since node $k$ is removed and since $\mathcal{C}(r,[K])$ is an $r$-balanced scheme, the subfiles that were not originally stored in node $k$ are still replicated $r$ times among the surviving nodes. However, each of the subfiles originally available in node $k$ is now available at only $(r-1)$ of the remaining $(K-1)$ nodes. Let $\mathcal{A}_{k}$ denote the index set of these subfiles, i.e., $\mathcal{A}_{k}=\{\boldsymbol{i} \in \mathcal{S}([K], K-r) \mid k \notin$ $i\}$.

We partition the set of subfiles $\left\{W_{i} \mid \boldsymbol{i} \in \mathcal{A}_{k}\right\}$, into groups, each of which will be coded and communicated together for data rebalancing. The groups are indexed by length $(K-1-r)$ vectors $\boldsymbol{i}^{\prime} \in \mathcal{S}([K], K-1-r)$ where $k \notin \boldsymbol{i}^{\prime}$, i.e., the vectors $\boldsymbol{i}^{\prime} \in \mathcal{S}(\overline{\mathcal{K}}, K-1-r)$. For each such $\boldsymbol{i}^{\prime}$, we define

$$
\mathcal{G}_{\boldsymbol{i}^{\prime}}=\left\{\boldsymbol{i} \in \mathcal{A}_{k} \mid\left[\begin{array}{llll}
i_{2} & i_{3} & \cdots & i_{K-r}
\end{array}\right]=\boldsymbol{i}^{\prime}\right\} .
$$

The number of vectors $\boldsymbol{i}$ such that $\left[\begin{array}{lll}i_{2} & \cdots & i_{K-r}\end{array}\right]=\boldsymbol{i}^{\prime}$ and $\boldsymbol{i} \in \mathcal{A}_{k}$, i.e., the number of choices for the component $i_{1}$ such that $i_{1} \notin \boldsymbol{i}^{\prime}$ and $i_{1} \neq k$, is $r$. We also observe that for any two distinct $\boldsymbol{i}^{\prime}, \boldsymbol{j}^{\prime} \in \mathcal{S}(\overline{\mathcal{K}}, K-1-r)$, the sets $\mathcal{G}_{\boldsymbol{i}^{\prime}}$ and $\mathcal{G}_{\boldsymbol{j}^{\prime}}$ are non-intersecting. Thus, these $r$-sized groups form a partition of $\mathcal{A}_{k}$. Since $\left|\mathcal{A}_{k}\right|=P(K-1, K-r)$, we conclude that the number of such groups is $P(K-1, K-r) / r$.

The objective of our data rebalancing scheme is to replicate $W_{i}, \boldsymbol{i} \in \mathcal{A}_{k}$, at the storage node with index $i_{1}$. Note that since $i_{1} \in \boldsymbol{i}$, this subfile was not originally present in the node $i_{1}$. We achieve this objective by running one round of data exchange protocol for each of the $P(K-1, K-1-r)$ groups of subfiles, resulting in as many rounds of the protocol. Now, consider the subfiles $W_{\boldsymbol{i}}, \boldsymbol{i} \in \mathcal{G}_{\boldsymbol{i}^{\prime}}$, belonging to one of these groups. Let $p_{1}, \ldots, p_{r}$ be such that $\mathcal{G}_{\boldsymbol{i}^{\prime}}=\left\{\left[p_{1} \boldsymbol{i}^{\prime}\right], \cdots,\left[p_{r} \boldsymbol{i}^{\prime}\right]\right\}$. Using the facts that $p_{1}, \ldots, p_{r} \notin \boldsymbol{i}^{\prime}$ and $p_{1}, \ldots, p_{r} \neq k$, we observe that the subfile $\left.W_{\left[p_{1}\right.} \boldsymbol{i}^{\prime}\right]$ is available at the nodes $p_{2}, \ldots, p_{r}$, and we desire to replicate this subfile at node $p_{1}$. In all, each of the $r$ subfiles $W_{\left[p_{1} \boldsymbol{i}^{\prime}\right]}, \ldots, W_{\left[p_{r} \boldsymbol{i}^{\prime}\right]}$ is available in a unique $(r-1)$ sized subset of the $r$ storage nodes $p_{1}, \ldots, p_{r}$, and is required to be replicated at the remaining node as well. We can achieve the replication of the subfiles $W_{\left[p_{1} i^{\prime}\right]}, \ldots, W_{\left[p_{r} i^{\prime}\right]}$ at nodes $p_{1}, \ldots, p_{r}$, respectively, using the data exchange protocol [13] referred to in Section IV-C1. Since the size of each of the $r$ subfiles is $\ell=\frac{N}{P(K, K-r)}$ bits, the communication cost of the protocol is

$$
\frac{\ell r}{(r-1)}=\frac{N r}{(r-1) P(K, K-r)} \text { bits. }
$$

The above data exchange is performed for each group $\mathcal{G}_{i^{\prime}}$, $i^{\prime} \in \mathcal{S}(\overline{\mathcal{K}}, K-1-r)$. Since the number of groups is $P(K-$ $1, K-r) / r$, the overall communication cost of our rebalancing scheme is

$$
\frac{N P(K-1, K-r)}{(r-1) P(K, K-r)}=\frac{N r}{(r-1) K}=\frac{N \lambda}{(r-1)},
$$

and the resulting communication load is $L_{r e m}=1 /(r-1)$.

We now analyze the memory utilization at the surviving nodes at the end of rebalancing operation. Each node $m \neq$ $k$, has been originally storing subfiles with indices in the set $\mathcal{A}_{m}=\{\boldsymbol{i} \mid m \notin \boldsymbol{i}\}$, and will additionally store the subfiles corresponding to the indices $\left\{\boldsymbol{i} \in \mathcal{A}_{k} \mid i_{1}=m\right\}$. Thus, the number of subfiles in node $m$ after rebalancing is the sum of the sizes of these sets, which is

$P(K-1, K-r)+P(K-2, K-1-r)=K P(K-2, K-1-r)$.

Multiplying this by the size of each subfile, we obtain the overall size of the contents of node $m$ after rebalancing

$$
K P(K-2, K-1-r) \times \frac{N}{P(K, K-r)}=\frac{N r}{K-1} .
$$

Thus, $\lambda_{\text {rem }}=\frac{r}{(K-1)}=\lambda \frac{K}{(K-1)}$.

Our rebalancing scheme replicates each $W_{\boldsymbol{i}}, \boldsymbol{i} \in \mathcal{A}_{k}$, at exactly one of the surviving nodes $\overline{\mathcal{K}}$, increasing the replication factor of these subfiles among the nodes in $\overline{\mathcal{K}}$ from $(r-1)$ to $r$. The subfiles which were not contained in node $k$ in $\mathcal{C}(r,[K])$, already have a replication factor of $r$ among the nodes in $\overline{\mathcal{K}}$. Thus, we conclude that the achieved target database is an $r$ balanced database across $K-1$ nodes.

3) Re-indexing and Structural Invariance: We now combine the subfiles ( $K$ of them at a time) available in the nodes $\overline{\mathcal{K}}$ after rebalancing, and then re-index them. This re-indexing operation uses vectors from $\mathcal{S}(\overline{\mathcal{K}}, K-1-r)$, i.e., vectors $\boldsymbol{i}^{\prime}$ of length $K-1-r$ whose components are distinct elements of $\overline{\mathcal{K}}=[K] \backslash k$. Our objective is to re-index the subfiles such that each node $m \in \overline{\mathcal{K}}$ consists of all the re-indexed subfiles whose indices $\boldsymbol{i}^{\prime} \in \mathcal{S}(\overline{\mathcal{K}}, K-1-r)$ satisfy $m \notin \boldsymbol{i}^{\prime}$. Since $|\overline{\mathcal{K}}|=K-1$, this ensures that the new database is identical to $\mathcal{C}(r,[K-1])$, up to a relabelling of the storage nodes.

Consider any $\boldsymbol{i}^{\prime} \in \mathcal{S}(\overline{\mathcal{K}}, K-1-r)$. There exist distinct $j_{1}, \ldots, j_{r} \in \overline{\mathcal{K}}$ such that $j_{1}, \ldots, j_{r} \notin i^{\prime}$. Further, $k \notin \boldsymbol{i}^{\prime}$. Assuming $j_{1}<j_{2}<\cdots<j_{r}$, a new re-indexed subfile $W_{\boldsymbol{i}^{\prime}}$ 
is obtained by concatenating the contents of the following $K$ original subfiles (in that order) whose indices are

$$
\begin{aligned}
& {\left[\begin{array}{ll}
j_{1} & \boldsymbol{i}^{\prime}
\end{array}\right],\left[\begin{array}{lll}
j_{2} & \boldsymbol{i}^{\prime}
\end{array}\right], \cdots,\left[\begin{array}{ll}
j_{r} & \boldsymbol{i}^{\prime}
\end{array}\right],} \\
& {\left[\begin{array}{llllll}
k & i_{1}^{\prime} & \cdots & i_{K-1-r}^{\prime}
\end{array}\right],\left[\begin{array}{lllll}
i_{1}^{\prime} & k & i_{2}^{\prime} & \cdots & i_{K-1-r}^{\prime}
\end{array}\right], \cdots,} \\
& {\left[\begin{array}{lllll}
i_{1}^{\prime} & i_{2}^{\prime} & \cdots & i_{K-1-r}^{\prime}
\end{array}\right] .}
\end{aligned}
$$

Note that, after rebalancing, any node $m \notin \boldsymbol{i}^{\prime}, m \neq k$, stores all the above $K$ subfiles. While rebalancing delivers $W_{\left[\begin{array}{ll}m & \boldsymbol{i}^{\prime}\end{array}\right]}$ to node $m$, the other $(K-1)$ subfiles are already present in this node by the design of $\mathcal{C}(r,[K])$. Thus, for every $\boldsymbol{i}^{\prime} \in$ $\mathcal{S}(\overline{\mathcal{K}}, K-1-r)$ and every choice of $m \notin \boldsymbol{i}^{\prime}, m \in \overline{\mathcal{K}}$, node $m$ can perform this re-indexing operation and store the reindexed file $W_{\boldsymbol{i}^{\prime}}$ in its memory. It is straightforward to see that $W_{\boldsymbol{i}^{\prime}}, \boldsymbol{i}^{\prime} \in \mathcal{S}(\overline{\mathcal{K}}, K-1-r)$, form a partition of the file $W$, and that node $m$ stores $W_{\boldsymbol{i}^{\prime}}$ if and only if $m \notin \boldsymbol{i}^{\prime}$. Thus the rebalanced database is identical to $\mathcal{C}(r,[K-1])$.

4) Rebalancing Load: The communication load of our rebalancing scheme for the removal of any node $k$ is equal to $L_{r e m}=1 /(r-1)$, and communication load for node addition is $L_{a d d}=1$. We conclude that the rebalancing load for our scheme is $\frac{1}{(r-1)}+1$.

\section{Converse of TheOrem 1}

We first consider the node addition case. Noting the fact that the new node arrives without any stored information, it is clear that any rebalancing scheme for node addition must necessarily involve communicating $\lambda_{a d d} N$ bits to the new node. Hence $L_{a d d}\left(\mathcal{R}_{\mathcal{C}, \mathcal{C}^{\prime}}^{\prime}\right) \geq 1$ for any rebalancing scheme $\mathcal{R}^{\prime}$ and any initial and target databases $\mathcal{C}, \mathcal{C}^{\prime}$.

We now obtain the converse for the case when there is one failed node (the node $K$, without loss of generality) among the nodes $[K]$. The proof of the converse proceeds quite similar to the proof of converse in [10] in the context of distributed computing with coded data shuffling (Section VI in [10]).

We assume that the file $W$ is a uniform random variable taking values from $\mathbb{F}_{2}^{N}$. For $k \in[K]$, let $\tilde{C}_{k}$ denote set of all bits of $C_{K}$ which are available in the storage of node $k$ in the initial database. For a subset $S \subseteq[K]$, let $\tilde{C}_{S}=\bigcup_{k \in S} \tilde{C}_{k}$.

For a subset of bits $B \subseteq C_{K}$, a subset of surviving nodes $S \subseteq[K-1]$, and some $j \in\{0,1, \ldots,|S|\}$, let $a_{B}^{j, S}$ denote the number of distinct bits of $B$ which are available in exactly $j$ of the nodes in $S$, and not anywhere else, i.e.,

$$
a_{B}^{j, S}=\sum_{\mathcal{J} \in\left(\begin{array}{c}
S \\
j
\end{array}\right)}\left|\left(\left(\bigcap_{k \in \mathcal{J}} \tilde{C}_{k}\right) \cap B\right) \backslash\left(\bigcup_{k \in[K-1] \backslash \mathcal{J}} \tilde{C}_{k}\right)\right|
$$

where $\left(\begin{array}{c}S \\ j\end{array}\right)$ denotes the set of $j$-sized subsets of $S$.

Based on our assumptions regarding the system before and after the node failure, we have the following statements to be true.

$$
\begin{aligned}
a_{C_{K}}^{1,\{K\}} & =\left|C_{K}\right|=\lambda N . \\
\sum_{j=1}^{K} j a_{\{c\}}^{j,[K]} & =r, \forall c \in C_{K} . \\
\sum_{j=1}^{K} j a_{C_{K}}^{j,[K]} & =r \lambda N \\
\sum_{j=1}^{K-1} a_{C_{K}}^{j,[K-1]} & =\left|C_{K}\right|=\lambda N . \\
\sum_{j=1}^{K-1} j a_{\{c\}}^{j,[K-1]} & =r-1, \forall c \in C_{K} . \\
\sum_{j=1}^{K-1} j a_{C_{K}}^{j,[K-1]} & =(r-1) \lambda N
\end{aligned}
$$

Equation 3 holds by definition of $a_{B}^{j, S}$ and since the bits in $C_{K}$ are distinct. Equation (4) holds since each bit of $C_{K}$ is repeated precisely in $r$ nodes across the network, which leads to (5). Equation (6) holds because we assume $r \geq 2$ (otherwise rebalancing after node $K$ failure would be impossible). Also, (7) is true since exactly one repetition of bit $c \in C_{K}$ is unavailable after the failure of node $K$, and (7) leads to (8).

After the failure of node $K$, the surviving part of the database has replication factor $r-1$ for bits in $C_{K}$. We then want to design the rebalancing scheme such that target database has replication factor $r$ for bits of $C_{K}$ also. This means any rebalancing scheme should be designed so that each bit $c \in C_{K}$ is to be communicated to exactly one node in $[K-1]$ which does not already contain $c$. Recalling the fact that $2 \leq r \leq(K-1)$, we also note that we should have $K \geq 3$. We now formalize these aspects of any valid rebalancing scheme now.

Let $D_{k}: k \in[K-1]$ denote the set of bits of $C_{K}$ to be stored in the node $k \in[K-1]$ respectively, in the target database. For a subset $S \subseteq[K-1]$, we also denote $D_{S} \triangleq$ $\cup_{k \in S} D_{k}$. Based on our requirements, the following are true.

$$
\begin{aligned}
\bigcup_{k \in[K-1]} D_{k} & =D_{[K-1]}=C_{K} \\
D_{k} \cap D_{k^{\prime}} & =\phi, \forall \text { distinct } k, k^{\prime} \in[K-1], \\
D_{k} \cap \tilde{C}_{k} & =\phi, \forall k \in[K-1] .
\end{aligned}
$$

For $k \in[K-1]$, let $X_{k}$ denote the set of transmitted bits by node $k$ to perform the rebalancing. Note that since the messages to be exchanged are subsets of $C_{K}$, we thus have that $H\left(X_{k} \mid \tilde{C}_{k}\right)=0$. For a subset $S \subseteq[K-1]$, we denote $X_{S} \triangleq\left\{X_{k}: k \in S\right\}$. We then want,

$$
H\left(D_{k} \mid X_{[K-1] \backslash k, \tilde{C}_{k}}\right)=0 .
$$

For a subset $S \in[K-1]$, we define the quantity $Y_{S}$ as follows.

$$
Y_{S}=\left\{D_{k}: k \in S\right\} \cup\left\{\tilde{C}_{k}: k \in S\right\} .
$$


Following the technique in [10], we first prove a lower bound on the quantity $H\left(X_{S} \mid Y_{\bar{S}}\right)$, where $\bar{S}=[K-1] \backslash S$. The converse will then follow by substituting $S=[K-1]$. The lower bound on $H\left(X_{S} \mid Y_{\bar{S}}\right)$ is given by the following lemma.

Lemma 1. For $S \subseteq[K-1]$ such that $|S| \geq 2$, we have

$$
H\left(X_{S} \mid Y_{\bar{S}}\right) \geq \sum_{j=1}^{|S|-1} \frac{a_{D_{S}}^{j, S}}{j} .
$$

Proof: We prove the lemma by induction. First consider the base case when $|S|=2$, and without loss of generality let $S=\{1,2\}$. Then $a_{D_{1}}^{1,\{1,2\}}$, by definition and by 111 , must lie in node 2 only and nowhere else in the $K-1$ nodes. By a similar argument for $a_{D_{2}}^{1,\{1,2\}}$, we must have that $H\left(X_{S} \mid Y_{\bar{S}}\right) \geq$ $a_{D_{1}}^{1,\{1,2\}}+a_{D_{2}}^{1,\{1,2\}}=a_{D_{\{1,2\}}^{1,\{1,2\}}}^{1,}$, which proves the base case.

Now we assume that the statement holds for all subsets of $[K-1]$ of size $t=|S|-1$. We then want to show it for $S$. We have the following expressions.

$$
\begin{aligned}
H\left(X_{S} \mid Y_{\bar{S}}\right) & =\frac{1}{|S|} \sum_{k \in S} H\left(X_{S}, X_{k} \mid Y_{\bar{S}}\right) \\
& =\frac{1}{|S|} \sum_{k \in S}\left(H\left(X_{S} \mid X_{k}, Y_{\bar{S}}\right)+H\left(X_{k} \mid Y_{\bar{S}}\right)\right) \\
& =\frac{1}{|S|}\left(\sum_{k \in S} H\left(X_{S} \mid X_{k}, Y_{\bar{S}}\right)+H\left(X_{S} \mid Y_{\bar{S}}\right)\right) .
\end{aligned}
$$

By re-arranging the terms, we get

$$
\begin{aligned}
H\left(X_{S} \mid Y_{\bar{S}}\right) & =\frac{1}{t} \sum_{k \in S} H\left(X_{S} \mid X_{k}, Y_{\bar{S}}\right) \\
& \geq \frac{1}{t} \sum_{k \in S} H\left(X_{S} \mid X_{k}, \tilde{C}_{k}, Y_{\bar{S}}\right) \\
H\left(X_{S} \mid Y_{\bar{S}}\right) & \geq \frac{1}{t} \sum_{k \in S} H\left(X_{S} \mid \tilde{C}_{k}, Y_{\bar{S}}\right),
\end{aligned}
$$

where 12 follows as $H\left(X_{k} \mid \tilde{C}_{k}\right)=0$. Now, we have for any $k \in S$,

$$
\begin{aligned}
& H\left(X_{S}, D_{k} \mid \tilde{C}_{k}, Y_{\bar{S}}\right)=H\left(X_{S} \mid \tilde{C}_{k}, Y_{\bar{S}}\right)+H\left(D_{k} \mid X_{S}, \tilde{C}_{k}, Y_{\bar{S}}\right) \\
& \quad=H\left(X_{S} \mid \tilde{C}_{k}, Y_{\bar{S}}\right)=H\left(D_{k} \mid \tilde{C}_{k}, Y_{\bar{S}}\right)+H\left(X_{S} \mid D_{k}, \tilde{C}_{k}, Y_{\bar{S}}\right),
\end{aligned}
$$

where the second equality in (13) follows because $D_{k}$ is decodable given $X_{S}, Y_{\bar{S}}$.

We now reduce the two components of the last expression in (13) separately. Firstly, because $D_{k}$ and $D_{k^{\prime}}$ are independent (as the $D_{k}$ s form a partition of $C_{K}$ ), we also have

$$
H\left(D_{k}, D_{\bar{S}} \mid \tilde{C}_{k}, \tilde{C}_{\bar{S}}\right)=H\left(D_{k} \mid \tilde{C}_{k}, \tilde{C}_{\bar{S}}\right)+H\left(D_{\bar{S}} \mid \tilde{C}_{k}, \tilde{C}_{\bar{S}}\right)
$$

We now have the following expressions.

$$
\begin{aligned}
H\left(D_{k} \mid \tilde{C}_{k}, Y_{\bar{S}}\right) & =H\left(D_{k} \mid \tilde{C}_{k}, D_{\bar{S}}, \tilde{C}_{\bar{S}}\right) \\
& =H\left(D_{k} \mid \tilde{C}_{k}, \tilde{C}_{\bar{S}}\right), \\
& =H\left(D_{k} \mid \tilde{C}_{\bar{S} \cup\{k\}}\right),
\end{aligned}
$$

where (15) follows from (14). The expression in $(16)$ is the number of bits of $D_{k}$ which are present only in $S \backslash k$ (since every bit of $D_{k}$ must be present in at least one of the $K-1$ surviving nodes). Thus we have from (16),

$$
H\left(D_{k} \mid \tilde{C}_{k}, Y_{\bar{S}}\right)=H\left(D_{k} \mid \tilde{C}_{\bar{S} \cup\{k\}}\right)=\sum_{j=1}^{t} a_{D_{k}}^{j, S \backslash k} .
$$

We now bound the second term of the last expression of (13). We have the following.

$$
\begin{aligned}
H\left(X_{S} \mid D_{k}, \tilde{C}_{k}, Y_{\bar{S}}\right) & =H\left(X_{S} \mid Y_{\bar{S} \cup\{k\}}\right) \\
& =H\left(X_{S \backslash k} \mid Y_{\bar{S} \cup\{k\}}\right) \\
& \geq \sum_{j=1}^{t-1} \frac{a_{D_{S \backslash k}}^{j, S \backslash k}}{j},
\end{aligned}
$$

where the second equality follows because $Y_{\bar{S} \cup k}$ contains $\tilde{C}_{k}$ and $H\left(X_{k} \mid \tilde{C}_{k}\right)=0$, and the last inequality follows by the induction hypothesis. Now, by using (19) and (17) in (13), we get

$$
H\left(X_{S}, D_{k} \mid \tilde{C}_{k}, Y_{\bar{S}}\right) \geq \sum_{j=1}^{t} a_{D_{k}}^{j, S \backslash k}+\sum_{j=1}^{t-1} \frac{a_{D_{S \backslash k}}^{j, S \backslash k}}{j} .
$$

Now,

$\sum_{k \in S} a_{D_{k}}^{j, S \backslash k}$

$=\sum_{k \in S} \sum_{n=1}^{\left|D_{k}\right|} \mathbb{I}\left(n^{t h}\right.$ bit of $D_{k}$ is stored in exactly $j$ nodes of $\left.S \backslash k\right)$

$=\sum_{k \in S} \sum_{n=1}^{\left|D_{k}\right|} \mathbb{I}\left(n^{t h}\right.$ bit of $D_{k}$ is stored in exactly $j$ nodes of $\left.S\right)$

$=\sum_{k \in S} a_{D_{k}}^{j, S}=a_{D_{S}}^{j, S}$,

where the second equality holds because no bits of $D_{k}$ are in $\tilde{C}_{k}$. We have also the following,

$$
\begin{aligned}
\sum_{k \in S} a_{D_{S \backslash k}}^{j, S \backslash k} & =\sum_{k \in S}\left(a_{D_{S}}^{j, S \backslash k}-a_{D_{k}}^{j, S \backslash k}\right) \\
& =\sum_{k \in S} a_{D_{S}}^{j, S \backslash k}-a_{D_{S}}^{j, S},
\end{aligned}
$$


where the second equality follows from 21). Further,

$$
\begin{aligned}
& \sum_{k \in S} a_{D_{S}}^{j, S \backslash k} \\
& =\sum_{k \in S} \sum_{n=1}^{\left|D_{S}\right|} \mathbb{I}\left(n^{t h} \text { bit of } D_{S} \text { is stored in exactly } j \text { nodes of } S \backslash k\right) \\
& =\sum_{k \in S} \sum_{n=1}^{\left|D_{S}\right|}\left(\mathbb{I}\left(n^{t h} \text { bit of } D_{S} \text { is stored in exactly } j \text { nodes of } S\right)\right. \\
& =\sum_{n=1}^{\left|D_{S}\right|}\left(\times \mathbb{I}\left(n^{t h} \text { bit of } D_{S} \text { is not stored in node } k\right)\right) \\
& \quad \times \sum_{k \in S} \mathbb{I}\left(n^{t h} \text { bit of } D_{S} \text { is stored in exactly } j \text { nodes of } S\right) \\
& =a_{D_{S}}^{j, S}(|S|-j) .
\end{aligned}
$$

where the last equality is true because

$$
\mathbb{I}\left(n^{t h} \text { bit of } D_{S} \text { is stored in exactly } j \text { nodes of } S\right)=a_{D_{S}}^{j, S} \text {. }
$$

Using (21) 22, and (23) in 20, we get

$$
\begin{aligned}
\sum_{k \in S} H\left(X_{S}, D_{k} \mid \tilde{C}_{k}, Y_{\bar{S}}\right) & \geq \sum_{j=1}^{t} a_{D_{S}}^{j, S}+\sum_{j=1}^{t-1} \frac{a_{D_{S}}^{j, S}(|S|-1-j)}{j} \\
& \geq a_{D_{S}}^{t, S}+\sum_{j=1}^{t-1} \frac{t \cdot a_{D_{S}}^{j, S}}{j}
\end{aligned}
$$

By using (24) and the second equality of (13) in (12), we get

$$
H\left(X_{S} \mid Y_{\bar{S}}\right) \geq \frac{a_{D_{S}}^{t, S}}{t}+\sum_{j=1}^{t-1} \frac{a_{D_{S}}^{j, S}}{j}=\sum_{j=1}^{t} \frac{a_{D_{S}}^{j, S}}{j} .
$$

This completes the proof of the lemma.

By applying Lemma 1 to the set $S=[K-1]$ and noting that $a_{D_{[K-1]}^{j,[K-1]}}=a_{C_{K}}^{j,[K-1]}$, we have

$H\left(X_{[K-1]}\right) \geq \sum_{j=1}^{K-2} \frac{a_{C_{K}}^{j,[K-1]}}{j}=\sum_{j=1}^{K-2} \frac{a_{C_{K}}^{j,[K-1]}}{j}=\sum_{j=1}^{K-1} \frac{a_{C_{K}}^{j,[K-1]}}{j}$,

where the last equality holds as $a_{C_{K}}^{K-1,[K-1]}=0$ since $r \leq K-1$ and by (4). As $\frac{1}{j}$ is convex in $j$ and since $\sum_{j=1}^{K-1} a_{C_{K}}^{j,[K-1]}=\lambda N$ by $\sqrt{6}$, we thus have from the above last equation

$$
\begin{aligned}
H\left(X_{[K-1]}\right) & \geq \lambda N \sum_{j=1}^{K-1} \frac{a_{C_{K}}^{j,[K-1]}}{\lambda N} \cdot \frac{1}{j} \\
& \geq \frac{\lambda N}{\sum_{j=1}^{K-1} \frac{j a_{C_{K}}^{j, 1 K-1]}}{\lambda N}}=\frac{\lambda N}{r-1},
\end{aligned}
$$

where the last expression is true by (8). The converse for the rebalancing load under node removal is then complete by the definition of the load in this case.

By definition of the optimal load $L^{*}(r)$, we have therefore showed that the lower bound expression in Theorem 1 is true. This completes the converse proof.

\section{APPENDIX A}

REVIEW OF DATA EXCHANGE PROTOCOL

Without loss of generality, label the $r$ nodes as $1, \ldots, r$, respectively, and assume that each node $m \in[r]$ contains the files $B_{j}, j \in[r] \backslash\{m\}$. That is, the only file not available at node $m$ is $B_{m}$. The objective of the data exchange protocol is to deliver the file $B_{m}$ to node $m$ for each $m \in[r]$.

We split each file $B_{j}$ into $(r-1)$ parts and index the subfiles using the elements of the set $[r] \backslash\{j\}$, i.e., the file $B_{j}$ is partitioned into subfiles $B_{j, 1}, B_{j, 2}, \ldots, B_{j, j-1}, B_{j, j+1}, B_{j, j+2} \ldots, B_{j, r}$. We assume that each subfile is of size $\ell /(r-1)$. In the protocol, each node $i$ broadcasts the following coded packet

$$
E_{i}=\bigoplus_{j \neq i} B_{j, i}
$$

to all the other nodes, where $\oplus$ denotes binary XOR. Since each coded packet is of length $\ell /(r-1)$ and there are $r$ such transmissions, the overall communication cost is $\ell r /(r-1)$.

We now argue that these $r$ coded packets are sufficient for each node to meet its demand. Consider a node $m$ that demands $B_{m}$ and observes the coded packets $E_{i}, i \neq m$. Note that the subfiles of $B_{m}$ are $B_{m, i}, i \neq m$. Node $m$ decodes the subfile $B_{m, i}$ from $E_{i}$ as follows

$$
\begin{aligned}
E_{i} \bigoplus\left(\bigoplus_{j \neq m, i} B_{j, i}\right) & =\left(\bigoplus_{j \neq i} B_{j, i}\right) \bigoplus\left(\bigoplus_{j \neq m, i} B_{j, i}\right) \\
& =B_{m, i} .
\end{aligned}
$$

This decoding operation is possible since node $m$ knows the files $B_{j}, j \neq m$.

\section{REFERENCES}

[1] “Apache ignite," (Last accessed in 2019). [Online]. Available: https://ignite.apache.org/

[2] K. Shvachko, H. Kuang, S. Radia, and R. Chansler, "The hadoop distributed file system," in 2010 IEEE 26th Symposium on Mass Storage Systems and Technologies (MSST), May 2010, pp. 1-10.

[3] "Why hdfs data becomes unbalanced (hortonworks data platform documentation )," 2012 (Last accessed in 2019). [Online]. Available: https://docs.hortonworks.com/HDPDocuments/HDP3/HDP-3.1.0/ data-storage/content/why_hdfs_data_becomes_unbalanced.html

[4] "No shard left behind: dynamic work rebalancing in google cloud dataflow," (Last accessed in 2019). [Online]. Available: https://cloud.google.com/blog/products/gcp/ no-shard-left-behind-dynamic-work-rebalancing-in-google-cloud-dataflow

[5] "Data rebalancing in apache ignite (apache ignite documentation)," (Last accessed in 2019). [Online]. Available: https://apacheignite. readme.io/docs/rebalancing

[6] "Data rebalancing in apache hadoop (apache hadoop documentation)," (Last accessed in 2019). [Online]. Available: http://hadoop.apache.org/docs/current/hadoop-project-dist/ hadoop-hdfs/HdfsUserGuide.html\#Balancer

[7] "Rebalancing in ceph (ceph architecture)," (Last accessed in 2019). [Online]. Available: http://docs.ceph.com/docs/mimic/ architecture/\#rebalancing

[8] "The hadoop distributed file system: Architecture and design," (Last accessed in 2019). [Online]. Available: https://svn.eu.apache.org/repos/ asf/hadoop/common/tags/release-0.16.3/docs/hdfs_design.pdf

[9] M. A. Maddah-Ali and U. Niesen, "Fundamental limits of caching," IEEE Transactions on Information Theory, vol. 60, no. 5, pp. 28562867, May 2014. 
[10] S. Li, M. A. Maddah-Ali, Q. Yu, and A. S. Avestimehr, "A fundamental tradeoff between computation and communication in distributed computing," IEEE Transactions on Information Theory, vol. 64, no. 1, pp. 109-128, Jan 2018.

[11] K. Lee, M. Lam, R. Pedarsani, D. Papailiopoulos, and K. Ramchandran, "Speeding up distributed machine learning using codes," IEEE Transactions on Information Theory, vol. 64, no. 3, pp. 1514-1529, March 2018.

[12] K. V. Rashmi, N. B. Shah, and K. Ramchandran, "A piggybacking design framework for read-and download-efficient distributed storage codes," IEEE Transactions on Information Theory, vol. 63, no. 9, pp. 58025820, Sep. 2017.

[13] S. Li, M. A. Maddah-Ali, and A. S. Avestimehr, "Coded MapReduce," in 2015 53rd Annual Allerton Conference on Communication, Control, and Computing (Allerton), Sep. 2015, pp. 964-971. 